\title{
Genetic Analysis of Two Chicken Infectious Anemia Virus Variants-Related Gyrovirus in Stray Mice and Dogs: The First Report in China, 2015
}

\author{
Lichun Fang, Yang Li, Yixin Wang, Jiayuan Fu, Shuai Cui, Xiaohan Li, \\ Shuang Chang, and Peng Zhao \\ College of Animal Science and Veterinary Medicine, Shandong Agricultural University, Taian, China \\ Correspondence should be addressed to Shuang Chang; changshuang81@126.com and Peng Zhao; zhaopeng@sdau.edu.cn
}

Received 26 July 2016; Accepted 18 October 2016; Published 23 February 2017

Academic Editor: Nikolai V. Ravin

Copyright ( $) 2017$ Lichun Fang et al. This is an open access article distributed under the Creative Commons Attribution License, which permits unrestricted use, distribution, and reproduction in any medium, provided the original work is properly cited.

Chicken infectious anemia virus (CIAV) causes acute viral infection in chickens worldwide. It can infect chickens of all ages, but the disease is seen only in young chickens and is characterized by hemorrhagic lesions in the muscles, atrophic changes in the lymphoid organs, aplastic bone marrow, and immunosuppression causing increased mortality. Previous studies have demonstrated that CIAV can be isolated from blood specimens of humans and fecal samples of stray cats. In the present study, two variants of CIAV were isolated from fecal samples of mice (CIAV-Mouse) and stray dogs (CIAV-Dog), respectively. The genome of the two CIAV variants was sequenced and the results of the recombination detection program suggested that the CIAV-Dog strain could be a recombinant viral strain generated from parental CIAV strains, AB119448 and GD-1-12, with high confidence. Particularly, these findings were obtained from the comparison of genetic diversity and the relationship of CIAV between different hosts. This is the first report indicating that there is a significant difference in the number of transcription factor binding sites in CIAV noncoding regions from different hosts. Further studies are required to investigate the large geographic distribution of CIAV and monitor the variants, host range, and associated diseases.

\section{Introduction}

Chicken infectious anemia virus (CIAV) is an economically important pathogen affecting the poultry industry worldwide [1-7]; it was first isolated in Japan in 1979 by Yuasa [8]. The disease is characterized by atrophy of the bone marrow hematopoietic and lymphoid tissues (e.g., thymus) in young chickens, causing severe anemia and immunosuppression leading to increased mortality due to secondary complications [9]. The disease readily spreads horizontally, but vertical transmission appears to be the most important means of dissemination $[10,11]$. Horizontal transmission is caused by direct or indirect contact and is most likely to be transmitted orally and through the respiratory tract as well as feces from infected animals [7]. There is no strong evidence of CIAV posing a threat to the human health. However, existing evidence suggests that CIAV-related viruses can be found in feces of stray cats, and feces, blood, and skin of humans
[12-18], indicating that CIAV may be a potential threat to human health.

CIAV is a member of the genus Gyrovirus belonging to the family Circoviridae [3]. The virion of CIAV is nonenveloped, icosahedral, and approximately $25 \mathrm{~nm}$ in diameter, with a negative-sense, single-stranded, circular DNA genome, approximately 2298-2319 nucleotides in length [4]. The genome consists of three major partially overlapping open reading frames that encode peptides of 51.6 (VP1), 24 (VP2), and 13.6 (VP3) $\mathrm{kDa}$ [19]. The noncoding region of the CIAV genome is only $0.3 \mathrm{~kb}$, but it shows complete promoter activity and contains more than a dozen conserved sequences related to replication and transcriptional regulation [20].

CIAV isolates show extremely limited genetic variability worldwide [21]. Viruses belonging to the Circoviridae family are not well studied, and little is known about CIAV recombination events. Cases of CIAV recombination have been reported in chickens in China, and the only recombination 
region is located in the coding region of VP1 [22, 23]. Zhang et al. reported the first evidence of CIAV homologous recombination in cats [17].

In this study, two strains of CIAV from stray dogs and mice were isolated for the first time in an attempt to elucidate the mechanism of CIAV infection in various species. We analyzed the genetic diversity among different hosts of CIAV. The first evidence of CIAV homologous recombination in dogs is explored and an obvious difference in the number of transcription factor binding sites between different hosts was found.

\section{Materials and Methods}

2.1. Material Source. During the year 2015, fecal samples from 42 stray dogs and 50 mice were collected in Tai'an, Shandong.

2.2. DNA Extraction and CIAV Detection. DNA was extracted from fecal samples of 92 subjects using a commercial TIANamp Genomic DNA Kit (Tiangen Biotech Co., Ltd., China) according to the manufacturer's instructions. DNA extracted from all the samples was stored at $-20^{\circ} \mathrm{C}$ until further analysis. Primers were designed using Primer Premier 6.0 to amplify $842 \mathrm{bp}$ of the partial coding regions of CIAV. The forward primer was $5^{\prime}$ GCATTCCGAGTGGTTACTATTCC- $3^{\prime}$ and the reverse primer was $5^{\prime}$-CGTCTTGCCATCTTACAGTCTTAT- $3^{\prime}$. The PCR reaction was performed in a total volume of $25 \mu \mathrm{L}$ containing $2.5 \mu \mathrm{L}$ PCR buffer $\left(\mathrm{Mg}^{2+}\right), 2 \mu \mathrm{L}$ dNTPs $(2.5 \mathrm{mM}), 0.5 \mu \mathrm{L}$ of each primer, $17 \mu \mathrm{L}$ distilled water, $1 \mu \mathrm{L}$ template DNA, and $0.5 \mu \mathrm{L}$ rTaq DNA polymerase (TaKaRa, Biotechnology, Co. Ltd., Dalian, China). Amplification for CIAV was performed as follows: predenaturation at $95^{\circ} \mathrm{C}$ for $5 \mathrm{~min}$, followed by 30 cycles of denaturation at $95^{\circ} \mathrm{C}$ for $30 \mathrm{sec}$, annealing at $55^{\circ} \mathrm{C}$ for $50 \mathrm{sec}$, and extension at $72^{\circ} \mathrm{C}$ for $1 \mathrm{~min}$. PCR products of $842 \mathrm{bp}$ were analyzed using $1 \%$ agarose gel electrophoresis. Positive and negative controls were included in each PCR.

2.3. Virus Isolation. The two CIAV positive samples were propagated in Marek's disease virus-transformed MDCCMSB1 cell line. First, $0.5 \mathrm{~mL}$ virus suspension was mixed with the MSB1 cell pellet resuspended in $0.5 \mathrm{~mL}$ RPMI 1640 medium and incubated at $37^{\circ} \mathrm{C}$ for $1 \mathrm{~h}$. Then, $5 \mathrm{~mL}$ RPMI 1640 medium was added to the mixture, and the cells were suspended and incubated at $37^{\circ} \mathrm{C}$ for $3 \mathrm{~d}$. One-milliliter cell suspension was transferred to $4 \mathrm{~mL}$ fresh RPMI 1640 medium followed by incubation. Cells were repeatedly passaged and the virus was isolated.

2.4. Amplification, Cloning, and Sequencing of the CIAV Genome. Based on the CIAV sequences published in GenBank (Table 1), three pairs of primers were designed using DNAStar 7.0, and total DNA obtained in the previous step was amplified using PCR (Table 2). The amplified fragments were $843 \mathrm{bp}, 989 \mathrm{bp}$, and $802 \mathrm{bp}$ in length, to cover the entire CIAV genome. The PCR amplification was carried out in $50 \mu \mathrm{L}$ total volume containing $25-\mu \mathrm{L}$ buffer I, $16 \mu \mathrm{L}$ dNTPs,
$0.5 \mu \mathrm{L}$ of each primer, $13.5 \mu \mathrm{L}$ distilled water, $1 \mu \mathrm{L}$ DNA, and $0.5 \mu \mathrm{L}$ LA Taq polymerase (TaKaRa, Biotechnology, Dalian, China). All PCR amplification products were analyzed via agarose gel electrophoresis, followed by ethidium bromide staining. The PCR products were purified with a Gel Band Purification Kit (Omega Bio-Tek, USA) and cloned into the pMD19-T vector (TaKaRa Bio Inc., Japan) and sequenced using an ABI 3730 Sanger-based genetic analyzer (Carlsbad, CA, USA). Sequencing was performed in triplicate.

2.5. Whole Genome Sequence Alignment and Phylogenetic Analysis. To establish the genotypes and clusters of the sequenced CIAV variants in this study, phylogenetic analysis was performed based on the complete genome sequence. The complete nucleotide sequence of CIAV variants and reference sequences were obtained from GenBank (Table 1). The DNA sequences and amino acid sequences (VP1, VP2, and VP3) were assembled using DNAStar (version 7; Madison, WI, USA). Multiple-sequence alignment was performed using Clustal W (BioEdit version 7). A neighbor-joining (NJ) tree based on the full-length nucleic acid sequence was constructed using MEGA 5.1 program [24]. The robustness of the NJ tree was evaluated via a bootstrap analysis with 1000 replicates.

\subsection{Sequence Analysis of the CIAV Genome in Noncoding} Region. Based on the entire aligned genome, the homology of the noncoding region was analyzed using DNAStar software to compare the homology of CIAV with that of different reference strains. According to a previous report, CIAV binding factors and consensus sequences of the binding sites [20] in different hosts were searched and noted (Table 3).

2.7. Identification of CIAV Genomic Recombination. To detect recombinant patterns, parental strains, and potential putative recombination breakpoints in CIAV variants in this study, the recombination detection program 4 (RDP 4) was applied [25] using nine methods (RDP, GENECONV, BootScan, MaxChi, Chimaera, SiScan, Phyl- Pro, LARD, and 3Seq) with general settings (window size $=20$, highest multiple-comparisoncorrected $P$ value $=0.01$, Bonferroni correction, finding consensus daughter sequences, and polishing breakpoints). For the RDP algorithm, the reference sequence parameter (internal and external reference) was used as recommended in the manual.

To confirm the potential parental CIAV lineages and putative recombination breakpoints previously analyzed and estimated by RDP software, SIMPLOT software v. 3.5.1 [26] was employed to further investigate this possible recombination event.

\section{Results}

3.1. Detection of Viruses and Amplification of the Whole Genome by PCR. PCR analysis was performed on fecal samples from 92 subjects (42 stray dogs and 50 
TABLE 1: The GenBank accession numbers of full-length CIAV genomes in isolates from different hosts.

\begin{tabular}{|c|c|c|c|c|c|}
\hline Accession number & Strain name & Host & Year & Country (area) & Whole length \\
\hline M81223 & M81223 & Chicken & 1993 & Germany & $2298 \mathrm{bp}$ \\
\hline CAU65414 & Australia 704 & Chicken & 1996 & Australia & $2298 \mathrm{bp}$ \\
\hline CAU66304 & CAU66304 & Gallusgallus & 1997 & UK & $2319 \mathrm{bp}$ \\
\hline AB027470 & TR20 & Chicken & 1999 & Japan & $2298 \mathrm{bp}$ \\
\hline AB031296 & $\mathrm{A} 2$ & Chicken & 2000 & Japan & $2298 \mathrm{bp}$ \\
\hline AF313470 & AF313470 & Chicken & 2000 & USA & 2294 bp \\
\hline AF227982 & AF227982 & Chicken & 2001 & Australia & $2286 \mathrm{bp}$ \\
\hline AB046590 & AH9410 & Chicken & 2001 & Japan & $2298 \mathrm{bp}$ \\
\hline AF475908 & AF475908 & Chicken & 2002 & China & $2298 \mathrm{bp}$ \\
\hline AJ297685 & Clone 34 & Chicken & 2002 & Germany & $2297 \mathrm{bp}$ \\
\hline AF390102 & SMSC-1P60 & Chicken & 2003 & Malaysia & $2298 \mathrm{bp}$ \\
\hline AF285882 & SMSC-1 & Chicken & 2003 & Malaysia & $2298 \mathrm{bp}$ \\
\hline AF395114 & $\mathrm{BD}-3$ & Chicken & 2004 & Bangladesh & $2298 \mathrm{bp}$ \\
\hline DQ141673 & SD22 & Chicken & 2005 & China & 2298 bp \\
\hline DQ217401 & SMSC-1P123WT & Chicken & 2005 & Malaysia & $2298 \mathrm{bp}$ \\
\hline D10068 & CAE26P4 & Chicken & 2007 & Netherlands & 2298 bp \\
\hline EF683159 & 3711 & Chicken & 2007 & Australia & $2279 \mathrm{bp}$ \\
\hline DQ991394 & $01-4201$ & Chicken & 2007 & USA & $2298 \mathrm{bp}$ \\
\hline M55918 & Cuxhaven-1 & Chicken & 2008 & Netherlands & $2319 \mathrm{bp}$ \\
\hline FJ172347 & SDLY08 & Broiler chicken & 2008 & China & 2298 bp \\
\hline D31965 & CAECA123 & Chicken & 2008 & Japan & 2319 bp \\
\hline AB119448 & G6 & Chicken & 2009 & Japan & $2298 \mathrm{bp}$ \\
\hline AF311892 & 98D02152 & Chicken & 2010 & USA & $2298 \mathrm{bp}$ \\
\hline JX260426 & GD-1-12 & Chicken & 2012 & China & 2298 bp \\
\hline JX964755 & GXC060821 & Chicken & 2012 & China & $2292 \mathrm{bp}$ \\
\hline JQ690762 & China & Human & 2012 & China & $2316 \mathrm{bp}$ \\
\hline KF224935 & GD-K-12 & Chicken & 2013 & China & 2298 bp \\
\hline KJ872513 & CIAV-10 & Chicken & 2014 & Argentina & 2298 bp \\
\hline KM496307 & SC-MZ42A & Chicken & 2014 & China & 2298 bp \\
\hline NC001427 & Cux-1 & Chicken & 1991 & USA & $2319 \mathrm{bp}$ \\
\hline KC414026 & Cat-Gyv & Cat & 2014 & China & $2295 \mathrm{bp}$ \\
\hline JQ308210 & GyV3 & Human & 2011 & USA & $2359 \mathrm{bp}$ \\
\hline JX310702 & GyV4 & Human & 2012 & Hong Kong & 2034 bp \\
\hline KU645524 & CIAV-Dog & Dog & 2015 & China & $2298 \mathrm{bp}$ \\
\hline KU645525 & CIAV-Mouse & Mouse & 2015 & China & $2298 \mathrm{bp}$ \\
\hline
\end{tabular}

TABle 2: Primers used for genome amplification.

\begin{tabular}{llr}
\hline Primers & Sequence & Product length \\
\hline F1 & $5^{\prime}$-GCATTCCGAGTGGTTACTATTCC-3' & 843 bp \\
R1 & $5^{\prime}$-CGTCTTGCCATCTTACAGTCTTAT-3' \\
F2 & $5^{\prime}$-CGAGTACAGGGTAAGCGAGCTAAA-3' \\
R2 & $5^{\prime}$-TGCTATTCATGCAGCGGACTT-3' & $989 \mathrm{bp}$ \\
F3 & $5^{\prime}$-ACGAGCAACAGTACCCTGCTAT-3' \\
R3 & $5^{\prime}$-CTGTACATGCTCCACTCGTT-3' & 802 bp \\
\hline
\end{tabular}

mice); two variants of CIAV were confirmed and named as CIAV-Dog and CIAV-Mouse, respectively. The whole genome of CIAV was amplified using three sets of primers, and the genome was further analyzed using DNA sequencing.
3.2. Complete Sequence and Phylogenetic Analysis. The complete genome sequences of the two CIAV variants were submitted to GenBank, under the accession numbers KU645525 and KU645524. The length of the genome of both CIAVMouse and CIAV-Dog strains was $2298 \mathrm{bp}$. The whole 
TABLE 3: Transcription factor-binding sequence elements ${ }^{\mathrm{A}}$.

\begin{tabular}{lccc}
\hline Motif & Consensus sequence & CIAV sequence & Numbers of sites \\
\hline TATA box & GTATA(A/T)A(A/T) & TATATAT & 1 \\
CCAAT box & AGCCAAT & GGGCGG & 1 \\
SP1 site & GGGCGG & ACGTCA & 1 \\
ATF site & ACGTCA & TACGTCA & 5 \\
CREB site & (T/G)(T/A)CGTCA & GTGGTTA & 4 \\
Core element of the SV40 enhancer & GTGG(A/T)(A/T)(A/T) & GGGGGGGGGG & GGGGATTCCCC \\
Erythroid specific G-string & GGGGGGGGGG & CTATTC & 1 \\
NFKB + H2TF1 sites & GGGGATTCCCC & GAAAAGGGGGGGGGG & 1 \\
Lymphoid specific site & CTATTC & CACTAT & 1 \\
Pu Motif & 9 purines & GAAAGTGACTTTC \\
CACTAT & AT rich CACTAT & CGTTGCGAAAGT & 1 \\
PEA-1 site & GGAAGTGACTAAC & TGCCACTGTCGA \\
GT II site & G(G/C)TGTGGAA(A/T)GT & CAGCC & 1 \\
MLTF & GGCCACGTGACC & CATCC & 1 \\
CACCC site: & CACCC & AATAAA & 2 \\
Poly(A) signal & AATAAA &
\end{tabular}

${ }^{\mathrm{A}}$ Binding factors and consensus sequences of the binding sites are reviewed in references [29, 30].

genome sequence of these two strains was compared to that of other 35 strains from different countries and different hosts in GenBank (Table 1). According to the database, CIAV-Dog strain showed the highest homology (98.2\%) with SD22 strain (accession number DQ141673) isolated in Shandong, China, while it showed the lowest homology (40.4\%) with Hong Kong Human GyV4 strain (accession number JX310702). CIAV-Mouse strain showed the highest homology (98.3\%) with Australia 704 (accession number CAU65414) and Japan TR20 strains (accession number AB027470), while similar to CIAV-Dog, CIAV-Mouse showed the lowest homology (40.9\%) with Hong Kong Human GyV4 strain (accession number JX310702).

A phylogenetic analysis was performed using the two novel CIAV sequences and the 31 full-length genome sequences obtained from the public database based on the whole genome nucleotide sequence (Figure 1). As seen in Figure 1, the evolutionary tree can be divided into three groups of genes. These genes contain most of the CIAV strains in the first group, including the Cat-Gyv strain, which came from different hosts of different countries in different years. This group can be divided into 2 clusters. The China strain from humans was included in the first small cluster of branches, while the Cat-Gyv strain was included in the second small cluster of branches.

CIAV-Dog and SD22 strains isolated in 2005 were in the second group. The third group contained nine genomic sequences, including CIAV-Mouse. GD-K-12 strain was isolated in China in 2013. CIAV-10 strain was isolated from Argentina in 2014. TR20 and G6 strains were isolated in Japan in 1999 and 2008, respectively. The three strains (Australia 704, AF227982, and 3711) were isolated in Australia in 1996, 2001, and 2007, respectively. SMSC-1 was isolated in Malaysia in 2003. As stated above, the strains can be distinctly divided into 3 clusters in this group. The isolates from Australia (AF227982 and 3711) isolated in 2001 and 2007, respectively, were included in the first small cluster of branches; CIAVMouse and GD-K-12 isolated in 2013 were included in the second small cluster of branches, and the other five strains were included in the third small cluster of branches. The Chinese isolate, GD-K-12, isolated in 2013 had a lower growing ability and transmission capacity [2]. We speculated that CIAV-Mouse might also be an attenuated strain.

3.3. Sequence Analysis of CIAV Genome in Noncoding Region. As described in Table 3, we compared the noncoding region of the genome regulation related motif with that of other reference strains in different hosts (Figure 2).

The noncoding sequences in the CIAV genome were highly conservative (nucleotide homology 90.0-99.8\%), especially the transcription factor binding sites. These transcription factor binding sites from the CIAV in chickens were similar, but there existed an obvious difference in individual motifs among viruses from different hosts; compared to the CIAV in chicken, CIAV from other hosts lack the transcriptional binding site-related motif. The CIAV variants isolated from mice, cats, dogs, and humans do not contain CACTAT, PEA-1, and MLTF motifs, but the CIAV strains isolated from chicken contain all the three motifs (CACTAT, PEA-1, and MLTF). Interestingly, there are two poly(A) signals in all CIAV stemming from cats, dogs, and mice; whether there exist different terminations in the transcription process or they enhanced the stability of mRNA remains to be studied. All these differences can be seen in Figure 2.

3.4. Recombination Analysis. Recombination analysis identified 34 CIAV sequences from different hosts and detected one 


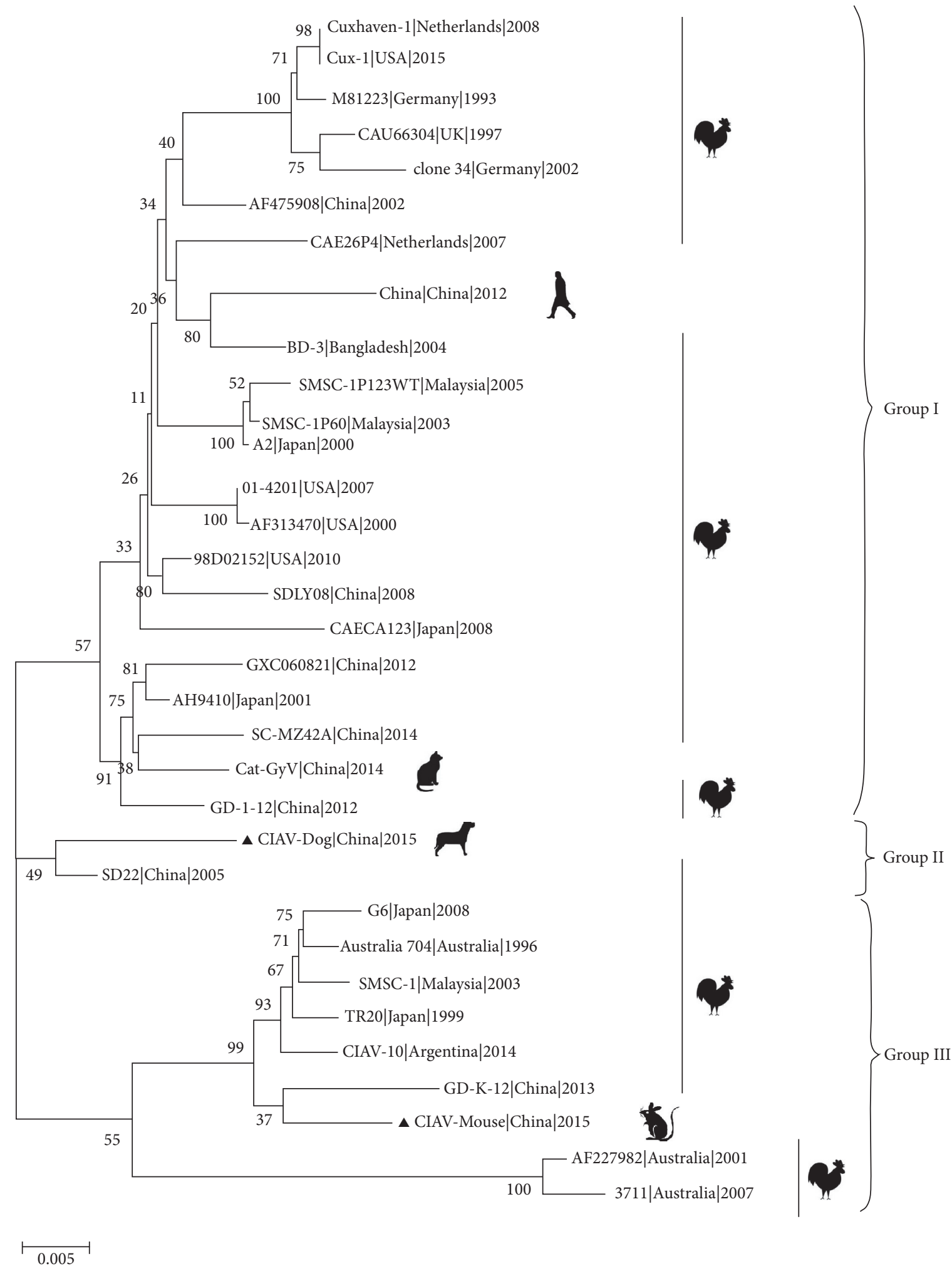

FIGURE 1: The molecular genetic evolution tree of CIAV strains based on the whole genome nucleotide sequence. Sequences from the present study are named as CIAV-Dog and CIAV-Mouse which are shown with a "black triangle." GenBank sequences were given the strains name followed by country name and time. The three major groups were identified as Group I, Group II, and Group III. The whole sequences were analyzed by using MEGA5.1 software with neighbor-joining (NJ) phylogenetic tree methods together with the novel sequence. Each tree was produced using a consensus of 1000 bootstrap replicates. 


\begin{tabular}{|c|c|c|c|c|c|}
\hline Motif & Chicken & Mouse & Cat & Dog & Human \\
\hline TATA box & 1 & 1 & 1 & 1 & 1 \\
\hline CCAAT box & 1 & 1 & 1 & 1 & 0 \\
\hline SP1 site & 1 & 1 & 1 & 1 & 1 \\
\hline ATF site & 5 & 5 & 4 & 5 & 5 \\
\hline CREB site & 4 & 4 & 3 & 4 & 4 \\
\hline Core element of the SV40 enhance & 1 & 1 & 1 & 1 & 1 \\
\hline Erythroid specific G-string & 1 & 1 & 0 & 1 & 1 \\
\hline $\mathrm{NFKB}+\mathrm{H} 2 \mathrm{TF} 1$ sites & 1 & 1 & 1 & 1 & 0 \\
\hline Lymphoid specific site & 1 & 1 & 1 & 1 & 1 \\
\hline Pu Motif & 1 & 1 & 0 & 1 & 1 \\
\hline CACTAT & 1 & 0 & 0 & 0 & 0 \\
\hline PEA-1 site & 1 & 0 & 0 & 0 & 0 \\
\hline GT II site & 1 & 1 & 1 & 1 & 1 \\
\hline MLTF & 1 & 0 & 0 & 0 & 0 \\
\hline \multicolumn{6}{|l|}{ CACCC site: } \\
\hline GAGCC & 2 & 2 & 2 & 2 & 1 \\
\hline CATCC & 2 & 1 & 1 & 1 & 2 \\
\hline Poly(A) signal & 1 & 2 & 2 & 2 & 1 \\
\hline
\end{tabular}

FIGURE 2: The number of transcription factor binding sites in noncoding regions of different hosts. Based on the whole genome that has been aligned. The transcription factor binding sites were searched and counted. The blue label means the motif numbers are same with chicken. The yellow label means deletion. The red label means addition.

TABLE 4: $P$ values of the recombinant calculated by different methods embedded in the RDP 4 software package.

\begin{tabular}{lc}
\hline Methods & Av. P Val \\
\hline RDP & $1.174 \times 10^{-04}$ \\
GENECONV & $2.323 \times 10^{-05}$ \\
BootScan & $2.262 \times 10^{-04}$ \\
MaxChi & $5.023 \times 10^{-05}$ \\
Chimaera & $2.014 \times 10^{-07}$ \\
SiScan & $1.220 \times 10^{-06}$ \\
PhyIPro & $\mathrm{n} / \mathrm{a}$ \\
LARD & $\mathrm{n} / \mathrm{a}$ \\
3Seq & $8.629 \times 10^{-09}$ \\
\hline
\end{tabular}

significant recombination event using the RDP 4 software. For this event, seven out of nine algorithms detected significant recombination at the same location in the CIAV genome with $P$ values ranging from $\left[8.629 \times 10^{-09}-1.174 \times 10^{-04}\right]$ (Table 4 ). The location of two significant break points was in the VP1 coding region (nt positions 1686 and 2122) of CIAVDog, which was considered as the daughter or recombinant, with the major parent being the GD-1-12 and the minor parent being AB119448.

In addition, to find proof for the results obtained using RDP software, a similarity plot analysis was carried out using SimPlot software. The analysis of the recombination was corroborative with the results of RDP software analysis. The results indicated that the recombinant exhibited a high nucleotide similarity with the isolates GD-1-12 (purple) and AB119448 (black) (Figure 3(a)). Bootscanning analysis also executed and confirmed the recombination event (Figure $3(\mathrm{~b}))$.

\section{Discussion}

CIAV was first reported by Yuasa et al., and since then the infection is found to be very common in chickens worldwide. In this study, two strains of CIAV from dogs and mice were first isolated, reported, and named as CIAV-Dog and CIAV-Mouse, respectively, and their whole genomes were 


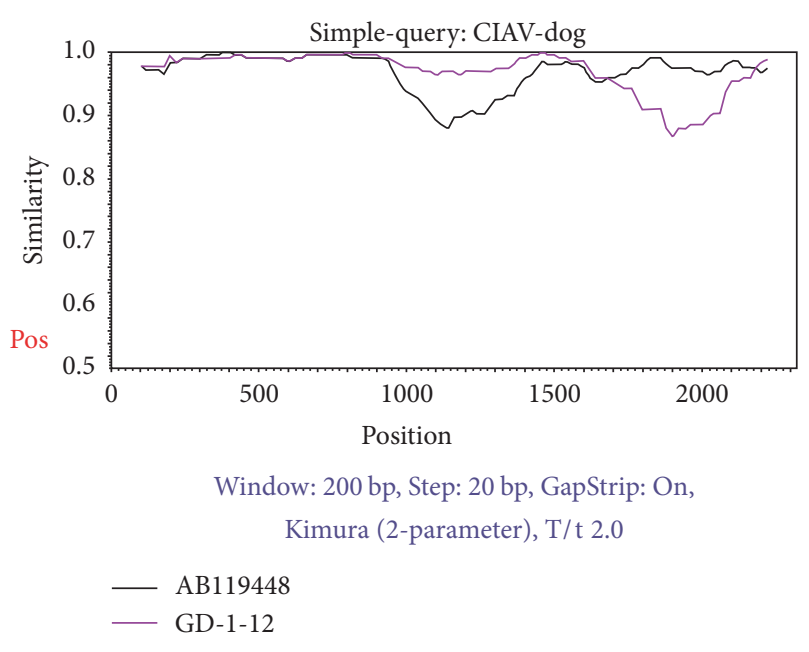

(a)

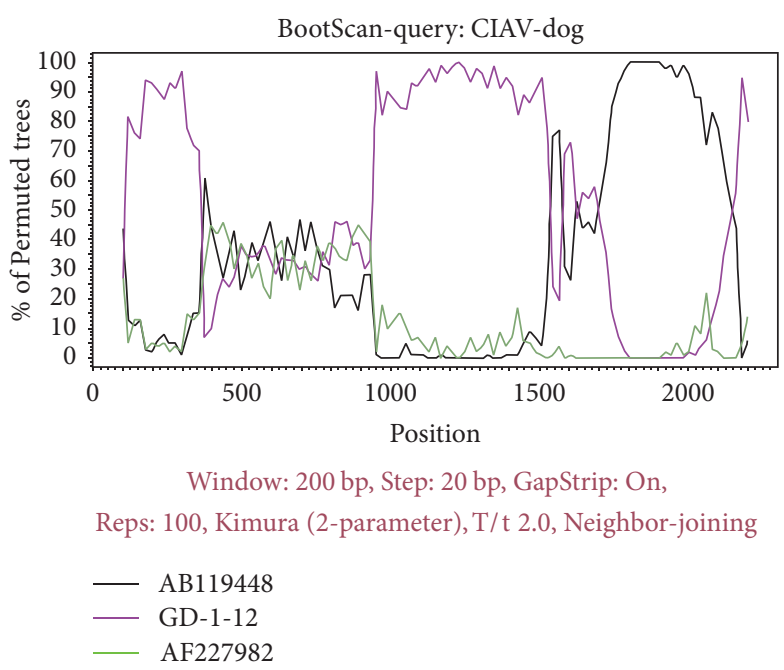

(b)

Figure 3: SimPlot recombination analysis. (a) Similarity plot generated using the complete genome of CIAV-Dog as query sequence, GD-1-12 (purple) and AB119448 (black) as two parent groups. Kimura 2-parameter was used as distance model with a transition-transversion ratio of 2. The $Y$-axis refers to percentage identity with a sliding window size of $200 \mathrm{bp}$ and with a step size of $20 \mathrm{bp}$ between plots. The $X$-axis represents the nucleotide positions in alignment. (b) Bootscanning analysis performed with CIAV-Dog as query sequence using a sliding window of $200 \mathrm{nt}$ moving in $20 \mathrm{nt}$ steps.

sequenced. A previous study showed that CIAV has been identified in two different hosts, including cats and humans [17]. A high prevalence of CIAV DNA was confirmed in diarrheal and normal feces from Chilean children [14]. Phan et al. reported that the high rate of codetection of three Gyroviruses (CIAV, AGV2/HGV, and GyV3) in human specimens might indicate common dietary exposure to foods that contain all the three viruses (e.g., chicken skin and meat) [14]. This may imply some uncertain dependency or interdependence on the proliferation of success. Although the results of this study found that CIAV variants were isolated from mouse and dog, they do not testify to the change of their natural host. All these variants share a common characteristic-a very high sequence similarity with CIAV from chickens. This finding indicates that the CIAV variant might have originated from CIAV-infected chickens. We know that these potential hosts are not the natural hosts of CIAV. However, it has been shown experimentally that the two hosts (dogs and mice) are carriers of CIAV. Davidson et al. also reported that feathers contribute to the horizontal transmission of CIAV, by carrying CIAV either on their surface or within the feather pulp [27]. The study has proved that dogs, mice, cats, or humans are unlikely to be involved in the epidemiology of CIAV. These potential hosts have the characteristics of mobility; thus they potentially boost CIAV transmission if they are carrying the virus. The results of this study revealed the expanded host range and rich genetic diversity of CIAV and highlighted the potential threat of CIAV to the health of mammals.

Moreover, we also tried to explore molecular characteristics of CIAV by analyzing the noncoding regions. The noncoding region of CIAV genome is only $0.3 \mathrm{~kb}$, but it showed a complete promoter activity [20]. In the corresponding expression vectors and specific cells, the complete noncoding region of CIAV genome can stimulate the expression of human growth hormone (hGH) gene [28]. In the noncoding region of CIAV, approximately 300 nucleotides are centrally distributed with more than a dozen conserved sequences related to replication and transcriptional regulation [20]. Whether these conserved sequences are necessary for the replication of the virus or for maintaining a balance between the virus and the specific cell is unclear and further studies are still required. In different hosts, the noncoding region sequences are highly conserved, but analysis of transcription factor binding sites shows obvious difference between the hosts (Figure 2). Whether the deletion or addition of the regulatory sites is due to the change in host or the mutation sites made it easier to spread to different hosts needs to be studied further. However, we assume that this obvious difference could be a way to estimate the variation among viruses in different hosts.

Natural recombination in VP1 of the CIAV genome, with a resultant new genotype, suggests a faster CIAV evolution [22]. A high mutation and gene recombination rate play an important role in the evolution of viruses, which is also the main cause of genetic diversity. In the process of restructuring, a large number of sequence changes can occur through genetic information exchange with other related viruses. Additionally, through the recombination analysis, there was a very high rate to support that CIAV-Dog might be a recombinant of AB119448 and GD-1-12 stemmed from chicken. Moreover, the recombination did not change the open reading frame. These results indicate that the CIAV variant might have originated from CIAV-infected chickens. Homologous recombination might cause a potential change in viral epidemiology. It is important to understand the extent 
of sequence variability in CIAV, to improve the management strategies to prevent CIAV infections in chickens, and to prevent the transmission from chickens to humans or other hosts. In view of the high infection rate of CIAV, the analysis of the pathogenicity of the recombinant virus in different hosts will be of great significance in future research.

In conclusion, we reported that chickens are the natural hosts of CIAV, but CIAV variants were detected in cats, dogs, mice, and humans. To explore the potential reason for increased transmission of CIAV, we should focus on the possibility of CIAV transmission by chickens, mice, cats, dogs, and humans. Considering the genetic evolution of CIAV strains, they have no apparent relationship with time and geographical area, and homologous recombination of CIAV can occur in different hosts. The present study noted that extensive surveillance of the virus in poultry, mammals, and other hosts should be carried out in the future.

\section{Competing Interests}

The authors declare that the research was conducted in the absence of any commercial or financial relationships that could be construed as a potential conflict of interests.

\section{Authors' Contributions}

Lichun Fang and Yang Li have contributed equally to this work.

\section{Acknowledgments}

Authors would like to thank Professor Zhizhong Cui at Shandong Agricultural University for his support. Authors are also grateful to Professor Weifeng Shi at Taishan Medical University for his technical assistance in recombinant analysis.

\section{References}

[1] W. Zhou, B. Yang, B. Shen, S. Han, and J. Zhou, "A serologic survey of antibody against chicken infectious anemia virus by indirect immunofluorescent assay in domestic poultry in China," Avian Diseases, vol. 40, no. 2, pp. 358-360, 1996.

[2] X. Zhang, Y. Liu, B. Wu et al., "Phylogenetic and molecular characterization of chicken anemia virus in southern China from 2011 to 2012," Scientific Reports, vol. 3, article 3519, 2013.

[3] S. Natesan, J. M. Kataria, K. Dhama, S. Rahul, and N. Bhardwaj, "Biological and molecular characterization of chicken anaemia virus isolates of Indian origin," Virus Research, vol. 118, no. 1-2, pp. 78-86, 2006.

[4] M. F. Ducatez, A. A. Owoade, J. O. Abiola, and C. P. Muller, "Molecular epidemiology of chicken anemia virus in Nigeria," Archives of Virology, vol. 151, no. 1, pp. 97-111, 2006.

[5] M. I. Craig, A. Rimondi, M. Delamer et al., "Molecular characterization of chicken infectious anemia virus circulating in Argentina during 2007," Avian Diseases, vol. 53, no. 3, pp. 331335, 2009.

[6] H.-R. Kim, Y.-K. Kwon, Y.-C. Bae, J.-K. Oem, and O.-S. Lee, "Molecular characterization of chicken infectious anemia viruses detected from breeder and broiler chickens in south korea," Poultry Science, vol. 89, no. 11, pp. 2426-2431, 2010.

[7] J. K. Rosenberger and S. S. Cloud, "The effects of age, route of exposure, and coinfection with infectious bursal disease virus on the pathogenicity and transmissibility of chicken anemia agent (CAA)," Avian Diseases, vol. 33, no. 4, pp. 753-759, 1989.

[8] N. Yuasa, T. Taniguchi, and I. Yoshida, "Isolation and some characteristics of an agent inducing anemia in chicks," Avian Diseases, vol. 23, no. 2, pp. 366-385, 1979.

[9] T. Taniguchi, N. Yuasa, M. Maeda, and T. Horiuchi, "Chronological observations on hemato-pathological changes in chicks inoculated with chicken anemia agent," National Institute of Animal Health Quarterly, vol. 23, no. 1, pp. 1-12, 1983.

[10] P. H. Jorgensen, L. Otte, O. L. Nielsen, and M. Bisgaard, "Influence of subclinical virus infections and other factors on broiler flock performance," British Poultry Science, vol. 36, no. 3, pp. 455-463, 1995.

[11] D. Todd, "Circoviruses: immunosuppressive threats to avian species: a review," Avian Pathology, vol. 29, no. 5, pp. 373-394, 2000.

[12] V. Sauvage, J. Cheval, V. Foulongne et al., "Identification of the first human Gyrovirus, a virus related to chicken anemia virus," Journal of Virology, vol. 85, no. 15, pp. 7948-7950, 2011.

[13] X. Zhang, Q. Xie, J. Ji et al., "Complete genome sequence analysis of a recent chicken anemia virus isolate and comparison with a chicken anemia virus isolate from human fecal samples in China," Journal of Virology, vol. 86, no. 19, pp. 10896-10897, 2012.

[14] T. G. Phan, L. Li, M. G. O’Ryan et al., "A third gyrovirus species in human faeces," Journal of General Virology, vol. 93, no. 6, pp. 1356-1361, 2012.

[15] D. K. W. Chu, L. L. M. Poon, S. S. S. Chiu et al., "Characterization of a novel gyrovirus in human stool and chicken meat," Journal of Clinical Virology, vol. 55, no. 3, pp. 209-213, 2012.

[16] F. Maggi, L. Macera, D. Focosi et al., "Human gyrovirus DNA in human blood, Italy," Emerging Infectious Diseases, vol. 18, no. 6, pp. 956-959, 2012.

[17] X. Zhang, Y. Liu, J. Ji et al., "Identification of a chicken anemia virus variant-related Gyrovirusin stray cats in China, 2012," BioMed Research International, vol. 2014, Article ID 313252, 7 pages, 2014.

[18] J. Ye, X. Tian, Q. Xie et al., "Avian gyrovirus 2 DNA in fowl from live poultry markets and in healthy humans, China," Emerging Infectious Diseases, vol. 21, no. 8, pp. 1486-1488, 2015.

[19] M. H. M. Noteborn, G. F. de Boer, D. J. van Roozelaar et al., "Characterization of cloned chicken anemia virus DNA that contains all elements for the infectious replication cycle," Journal of Virology, vol. 65, no. 6, pp. 3131-3139, 1991.

[20] B. M. Meehan, D. Todd, J. L. Creelan, J. A. P. Earle, E. M. Hoey, and M. S. McNulty, "Characterization of viral DNAs from cells infected with chicken anaemia agent: sequence analysis of the cloned replicative form and transfection capabilities of cloned genome fragments," Archives of Virology, vol. 124, no. 3-4, pp. 301-319, 1992.

[21] V. L. Van Santen, L. Li, F. J. Hoerr, and L. H. Lauerman, "Genetic characterization of chicken anemia virus from commercial broiler chickens in Alabama," Avian Diseases, vol. 45, no. 2, pp. 373-388, 2001.

[22] C.-Q. He, N.-Z. Ding, W. Fan, Y.-H. Wu, J.-P. Li, and Y.-L. Li, "Identification of chicken anemia virus putative intergenotype recombinants," Virology, vol. 366, no. 1, pp. 1-7, 2007. 
[23] Y. M. Eltahir, K. Qian, W. Jin, and A. Qin, "Analysis of chicken anemia virus genome: evidence of intersubtype recombination," Virology Journal, vol. 8, article 512, 2011.

[24] K. Tamura, D. Peterson, N. Peterson, G. Stecher, M. Nei, and S. Kumar, "MEGA5: molecular evolutionary genetics analysis using maximum likelihood, evolutionary distance, and maximum parsimony methods," Molecular Biology and Evolution, vol. 28, no. 10, pp. 2731-2739, 2011

[25] D. P. Martin, P. Lemey, M. Lott, V. Moulton, D. Posada, and P. Lefeuvre, "RDP3: a flexible and fast computer program for analyzing recombination," Bioinformatics, vol. 26, no. 19, pp. 2462-2463, 2010.

[26] K. S. Lole, R. C. Bollinger, R. S. Paranjape et al., "Fulllength human immunodeficiency virus type 1 genomes from subtype C-infected seroconverters in India, with evidence of intersubtype recombination," Journal of Virology, vol. 73, no. 1, pp. 152-160, 1999.

[27] I. Davidson, N. Artzi, I. Shkoda, A. Lublin, E. Loeb, and K. A. Schat, "The contribution of feathers in the spread of chicken anemia virus," Virus Research, vol. 132, no. 1-2, pp. 152-159, 2008.

[28] A. J. Douglas, K. Phenix, K. A. Mawhinney, D. Todd, D. P. Mackie, and W. L. Curran, "Identification of a $24 \mathrm{kDa}$ protein expressed by chicken anaemia virus," Journal of General Virology, vol. 76, no. 7, pp. 1557-1562, 1995.

[29] P. F. Johnson and S. L. McKnight, "Eukaryotic transcriptional regulatory proteins," Annual Review of Biochemistry, vol. 58, pp. 799-839, 1989.

[30] N. Jones, "Structure and function of transcription factors," Seminars in Cancer Biology, vol. 1, pp. 5-19, 1990. 

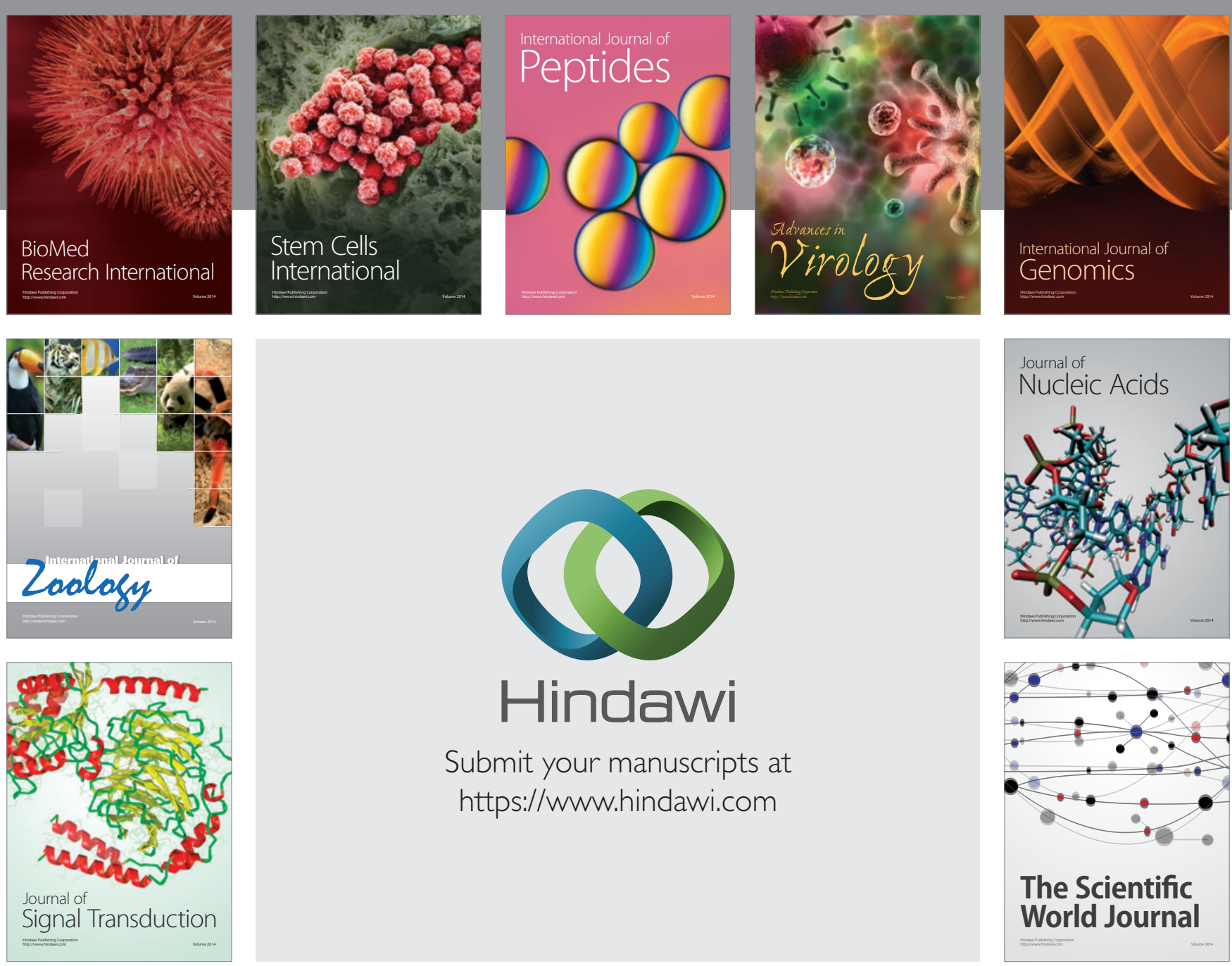

Submit your manuscripts at

https://www.hindawi.com
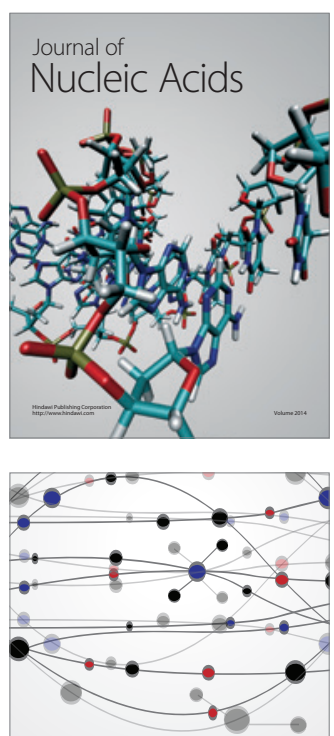

The Scientific World Journal
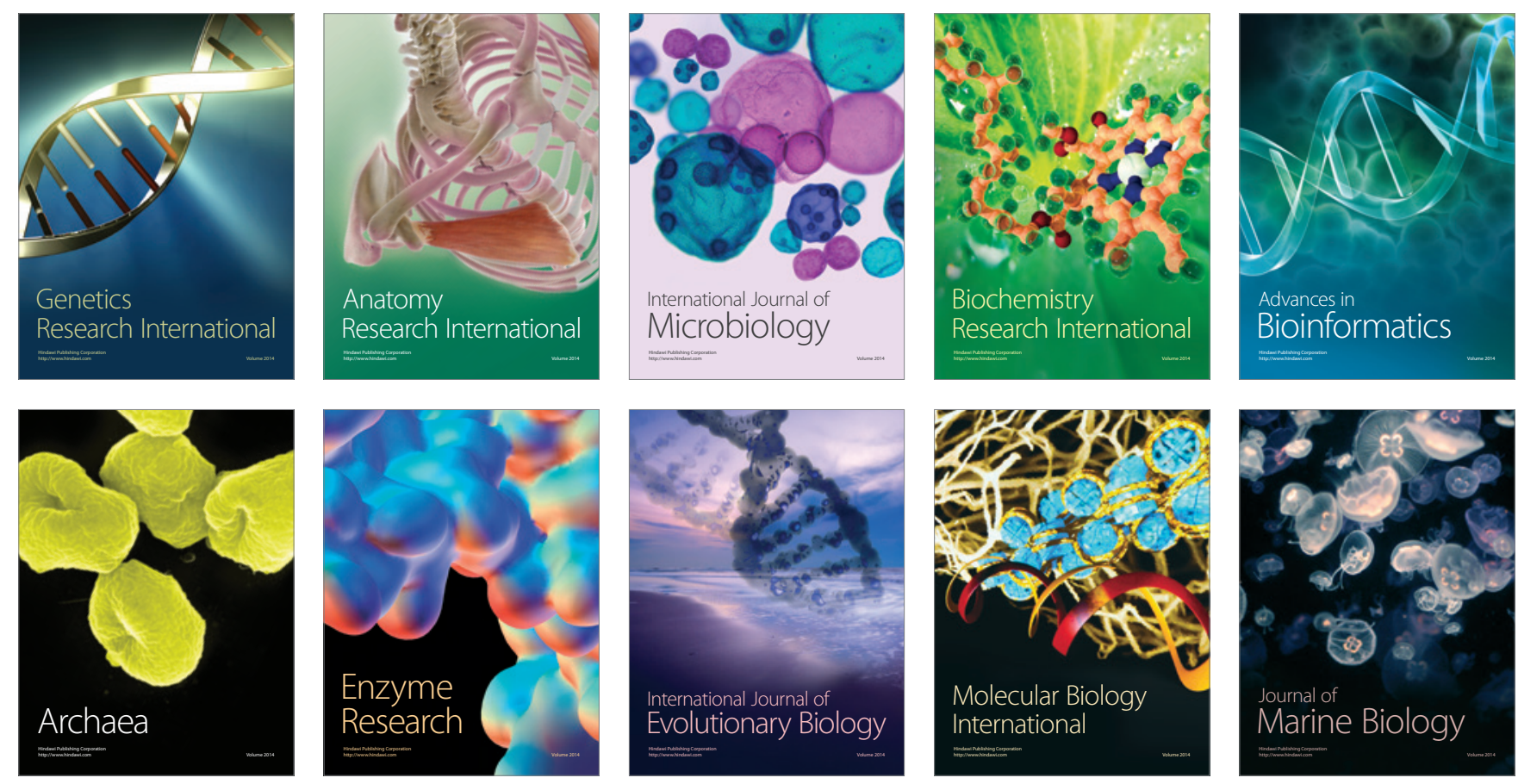